\title{
Optimising Partial-Order Plans Via Action Reinstantiation
}

\author{
Max Waters, Lin Padgham and Sebastian Sardina \\ RMIT University, Melbourne, Victoria 3000, Australia \\ \{max.waters, lin.padgham, sebastian.sardina\}@rmit.edu.au
}

\begin{abstract}
This work investigates the problem of optimising a partial-order plan's (POP) flexibility through the simultaneous transformation of its action ordering and variable binding constraints. While the former has been extensively studied through the notions of deordering and reordering, the latter has received much less attention. We show that a plan's variable bindings are often related to resource usage and their reinstantiation can yield more flexible plans. To do so, we extend existing POP optimality criteria to support variable reinstantiation, and prove that checking if a plan can be optimised further is NP-complete. We also propose a MAXSAT-based technique for increasing plan flexibility and provide a thorough experimental evaluation that suggests that there are benefits in action reinstantiation.
\end{abstract}

\section{Introduction}

This work investigates the modification of objects and resources in a plan to achieve higher flexibility w.r.t. action orderings. While the optimisation of action ordering has been extensively studied through the notions of plan deordering and plan reordering, both from a theoretical [Bäckström, 1998; Aghighi and Bäckström, 2017] and practical [Kambhampati and Kedar, 1994; Muise et al., 2016; Siddiqui and Haslum, 2012; Say et al., 2016] perspective, the optimisation of resources (or actions' domain objects) that are used in the course of executing a plan has received much less attention.

Ultimately, the aim is to achieve a least commitment approach to planning [Weld, 1994] that maximises executiontime flexibility by delaying decisions regarding action ordering and resource utilisation for as long as possible. Consider a planning instance from (a reduced version of) the IPC rovers domain, in which a fleet of rovers must navigate the surface of a planet, collecting soil and rock samples. The domain objects comprise two rovers $\left(R_{1}\right.$ and $\left.R_{2}\right)$ and three waypoints $\left(W_{1}, W_{2}\right.$, and $\left.W_{3}\right)$. There are soil and rock samples at waypoints $W_{2}$ and $W_{3}$, resp., and both rovers begin at $W_{1}$. The goal is to gather both samples. Plan $P_{1}$ in Figure 1a is an optimal solution: $R_{1}$ navigates from $W_{1}$ to $W_{2}$ and collects the soil sample, then navigates to $W_{3}$ and collects the rock sample. An examination of the causal structure of $P_{1}$ shows that

\author{
1. move $_{1}\left(\mathrm{R}_{1}, \mathrm{~W}_{1}, \mathrm{~W}_{2}\right)$ \\ 2. get-soil $\left(\mathrm{R}_{1}, \mathrm{~W}_{2}\right)$ \\ 3. move $\left(\mathrm{R}_{1}, \mathrm{~W}_{2}, \mathrm{~W}_{3}\right)$ \\ 4. get-rock $\left(\mathrm{R}_{1}, \mathrm{~W}_{3}\right)$
}

(a) Plan $P_{1}$
1. move $_{1}\left(\mathrm{R}_{1}, \mathrm{~W}_{1}, \mathrm{~W}_{2}\right)$

3. move $_{2}\left(\mathrm{R}_{2}, \mathrm{~W}_{1}, \mathrm{~W}_{3}\right)$

4. get-rock $\left(\mathrm{R}_{2}, \mathrm{~W}_{3}\right)$
2. get-soil $\left(\mathrm{R}_{1}, \mathrm{~W}_{2}\right)$

$$
\begin{aligned}
& O=\left\{\text { move }_{1}\left(x_{1}, x_{2}, x_{3}\right) \quad \theta=\left\{x_{1} \backslash \mathrm{R}_{1}, x_{2} \backslash \mathrm{W}_{1}, x_{3} \backslash \mathrm{W}_{2}\right.\right. \\
& \text { get-soil }\left(x_{4}, x_{5}\right) \quad x_{4} \backslash \mathrm{R}_{1}, x_{5} \backslash \mathrm{W}_{2} \\
& \text { move }_{2}\left(x_{6}, x_{7}, x_{8}\right) \quad x_{6} \backslash \mathrm{R}_{2}, x_{7} \backslash \mathrm{W}_{1}, x_{8} \backslash \mathrm{W}_{3} \\
& \left.\left.\operatorname{get} \text {-rock }\left(x_{9}, x_{10}\right)\right\} \quad x_{9} \backslash \mathrm{R}_{2}, x_{10} \backslash \mathrm{W}_{3}\right\} \\
& \prec=\left\{\text { move }_{1} \prec \text { get-soil, } \text { move }_{2} \prec \text { get-rock }\right\}
\end{aligned}
$$

(c) Partial-order plan $P_{3}$.

Figure 1: Three plans from the rovers domain. Subscripts have been used to better distinguish between actions of the same type.

the order of its actions cannot be altered without compromising its validity (e.g., step 3 threatens the causal link between steps 1 and 2 , as it moves $\mathrm{R}_{1}$ away from $\mathrm{W}_{2}$ ).

Plan $P_{2}$ in Figure $1 \mathrm{~b}$ is a modification of $P_{1}$ that instead uses rover $\mathrm{R}_{2}$ to collect the rock sample. This modification allows the plan's ordering constraints to be relaxed: the actions can be executed in any order so long as $1 \prec 2$ and $3 \prec 4$.

This simple example shows that an over-commitment to a particular set of resources (in this case, $\mathrm{R}_{1}$ ) can reduce a plan's possible orderings. Thus, this paper introduces the notions of reinstantiated deorderings and reinstantiated reorderings, namely, transformations of a plan under which ordering constraints can be removed or arbitrarily modified, resp., and the plan's actions' variable bindings can be changed. The idea is to seek alternative variable bindings supporting greater minimisation of the plan's ordering constraints than is possible if the bindings remained unchanged.

More concretely, we define optimality criteria for partialorder plans (POP) that take variable bindings into consideration (Section 3) and propose a technique for computing optimally reinstantiated POPs by encoding the problem into a MAXSAT instance (Section 4). Finally, we experimentally assess the benefits of reinstantiation by comparing it with existing de/reordering techniques (Section 5). Results show that optimising both bindings and orderings incurs a much greater computational cost than optimising orderings alone. However, when (even non-optimal) reinstantiated de/reorderings can be found, they are significantly more flexible than plans produced by standard optimisation methods. 


\section{Preliminaries}

\subsection{Logical Preliminaries}

The logical structures will be expressed in a function-free first-order language $\mathcal{L}$ with finitely many variable, constant, and predicate symbols, and the usual logical connectives, including equality and precedence [Brachman and Levesque, 2004, Chapter 2]. The letters $x, y$ and $z$ indicate variables, $c$ constants and $t, u$ and $v$ terms (all possibly with annotations). The notation $\vec{t}$ denotes ordered lists of possibly nonunique terms, with $\vec{t} i i]$ indicating the $i$-th element of the list and $\overrightarrow{t_{1}}=\overrightarrow{t_{2}}$ being shorthand for $\left|\overrightarrow{t_{1}}\right|=\left|\overrightarrow{t_{2}}\right| \wedge \overrightarrow{t_{1}}[1]=$ $\overrightarrow{t_{2}}[1] \wedge \cdots \wedge \overrightarrow{t_{1}}\left[\left|\overrightarrow{t_{1}}\right|\right]=\overrightarrow{t_{2}}\left[\left|\overrightarrow{t_{2}}\right|\right]$. For any structure $\eta$ expressed in $\mathcal{L}$, vars $(\eta)$ and consts $(\eta)$ denote the variables and constants appearing in $\eta$, resp.

A substitution $\theta$ is a mapping from variables to terms, for example $\theta=\left\{x_{1} / t_{1}, \ldots, x_{n} / t_{n}\right\}$ maps each variable $x_{i}$ to term $t_{i}$, for $1 \leq i \leq n$, and every other variable to itself. We use domain $(\theta)$ to denote the set of variables explicitly mapped by substitution $\theta$, and $\theta(x)$ to denote the term corresponding to variable $x$ under $\theta$. The result of applying a substitution $\theta$ to a logical structure $\eta$ is written $\eta \theta$, and means to simultaneously replace every variable $x$ in $\eta$ with $\theta(x)$. A substitution $\theta$ is ground if every variable in its domain is mapped to a ground term, and is complete w.r.t. $\eta$ iff $\operatorname{vars}(\eta) \subseteq$ domain $(\theta)$.

\subsection{Classical Planning Formalism}

Classical planning tasks will be expressed in a standard firstorder STRIPS formalism [Lifschitz, 1987].

\section{Classical Planning Tasks}

A classical planning task is a tuple $\Pi=\left\langle\mathcal{C}, \mathcal{O}, s_{I}, s_{G}\right\rangle$, where $\mathcal{C}$ is a set of constants, $\mathcal{O}$ is a set of operators, and $s_{I}$ and $s_{G}$ are sets of ground literals describing the initial state and goal, respectively. An operator is a tuple $o=\langle\operatorname{name}(o), \operatorname{vars}(o), \operatorname{pre}(o), \operatorname{post}(o)\rangle$, where name $(o)$ is the name, or type of the operator, vars $(o)$ is a list of variables and pre $(o)$ and post $(o)$ are finite sets of (ground or nonground) literals with variables taken from vars $(o)$. It will be assumed that for all operators, name $(o)=o$, and so operators will be referred to by name. When distinguishing between different operators of the same name, the notation $o(\vec{x})$ is used, where $\operatorname{vars}(o)=\vec{x}$. An action is a ground operator $a=\langle\operatorname{pre}(a)$, post $(a)\rangle$, where $\operatorname{pre}(a)$ and post $(a)$ are finite sets of ground literals. If $o$ is an operator and substitution $\theta$ is ground and complete w.r.t. $\operatorname{vars}(o)$, then $\langle\operatorname{pre}(o) \theta, \operatorname{post}(o) \theta\rangle$ is the action resulting from instantiating $o$ with $\theta$.

\section{Classical and Partial-Order Plans}

A classical plan $\vec{a}$ is a finite sequence of actions. Assuming that no variable appears in more than one operator, a plan can also be represented as $\vec{\partial} \theta$, where $\vec{O}$ is a list of operators and $\theta$ is a ground substitution that is complete w.r.t. $\vec{o}$. A planning task can be "embedded" into a plan by including the distinguished actions $a_{I}$ and $a_{G}$ whose pre/postconditions are the task's initial state and goal, resp. In this form, a classical plan is valid iff it is executable (i.e., the actions can be applied in sequence without violating their precondition requirements).
A partial-order plan (POP) is a generalised classical plan that need not completely define the order of its operators:

Definition 1. A partial-order plan $(P O P)$ is a tuple $P=$ $\langle O, \theta, \prec\rangle$ where $O$ is a set of operators, $\theta$ is a ground substitution which is complete w.r.t. to $O$, and $\prec$ is a strict, transitively closed partial order over $O$.

As with classical plans, a planning task is "embedded" into a POP via the inclusion of the distinguished parameter-free operators $o_{I}$ (which yields the initial state) and $o_{G}$ (which checks the goals). A POP is a compact representation of a set of classical plans, known as its linearisations:

Definition 2. A classical plan $\left\langle o_{1}, \ldots, o_{n}\right\rangle \theta$ is a linearisation of $P O P P=\langle O, \theta, \prec\rangle$ iff $O=\left\{o_{1}, \ldots, o_{n}\right\}$ and $\prec \subseteq\left\{a_{i} \prec a_{j}: 1 \leq i<j \leq n\right\}$.

A POP's validity is defined w.r.t. its linearisations:

Definition 3. A POP is valid iff all of its linearisations are executable.

Classical plans are special cases of POPs, and any classical plan $\left\langle o_{1}, \ldots, o_{n}\right\rangle \theta$ can be expressed as an equivalent partialorder plan $\left\langle\left\{o_{1}, \ldots, o_{n}\right\}, \theta,\left\{a_{i} \prec a_{j}: 1 \leq i<j \leq n\right\}\right\rangle$.

\section{The Producer-Consumer-Threat Formalism}

The producer-consumer-threat formalism (PCT) [Bäckström, 1998] is typically used to describe a POP's causal structure by identifying which actions produce, consume or threaten which ground literals. Here, it describes how operators produce, consume or threaten (possibly non-ground) literals:

Definition 4. Let o be an operator and $q(\vec{t})$ a literal. Then:

$$
\begin{gathered}
\operatorname{prods}(o, q(\vec{t})) \stackrel{\text { def }}{=} q(\vec{t}) \in \operatorname{post}(o) . \\
\operatorname{cons}(o, q(\vec{t})) \stackrel{\text { def }}{=} q(\vec{t}) \in \operatorname{pre}(o) . \\
\operatorname{thrtns}(o, q(\vec{t})) \stackrel{\text { def }}{=} \neg q(\vec{t}) \in \operatorname{post}(o) .
\end{gathered}
$$

In the field of partial-order causal link planning (POCL), a PCT-based notion of POP validity is used that derives from the implied causal dependencies between a POP's operators [Weld, 1994], not the validity of its linearisations. A causal link associates a consumer, (i.e., an operator precondition), with a producer (i.e., an operator postcondition):

Definition 5. A causal link is a 4-tuple $\left\langle o_{p}, q(\vec{t}), o_{c}, q(\vec{u})\right\rangle$ s.t. $\operatorname{prods}\left(o_{p}, q(\vec{t})\right)$ and cons $\left(o_{c}, q(\vec{u})\right)$.

The consumer is supported by the causal link iff it is preceded by, and codesignated with, the producer (i.e., $\theta(\vec{t})=$ $\theta(\vec{u}))$. The causal link is threatened iff a codesignated threat (i.e., some $o_{t}, q(\vec{v})$ s.t. $\operatorname{thrtns}\left(o_{t}, q(\vec{v})\right)$ and $\theta(\vec{v})=\theta(\vec{u})$ ) can be ordered between the producer and consumer. A POP $P$ 's implicit unthreatened causal links are denoted $L_{P}$, and defined as follows:

Definition 6. The unthreatened causal links of a $P O P P=$ $\langle O, \theta, \prec\rangle$ is the set $L_{P}$ s.t. $\left\langle o_{p}, q(\vec{t}), o_{c}, q(\vec{u})\right\rangle \in L_{P}$ iff:

1. $o_{p} \prec o_{c}$,

2. $\theta(\vec{t})=\theta(\vec{u})$, and

3. for all $o_{t}, q(\vec{v})$ s.t. thrtns $\left(o_{t}, q(\vec{v})\right)$, either $\theta(\vec{u}) \neq \theta(\vec{v})$, $o_{t} \prec o_{p}$ or $o_{c} \prec o_{t}$. 
A POP $P$ is $P O C L$-valid iff every consumer is supported by an unthreatened causal link:

Definition 7. A POP $P=\langle O, \theta, \prec\rangle$ is POCL-valid iff for all $o_{c} \in O$ and $q(\vec{u})$ s.t. cons $\left(o_{c}, q(\vec{u})\right)$, there exist $o_{p} \in O$ and $q(\vec{t})$ s.t. $\left\langle o_{p}, q(\vec{t}), o_{c}, q(\vec{u})\right\rangle \in L_{P}$.

This notion of validity is stronger than that in Definition 3, meaning that a POP might not be POCL-valid despite its linearisations being executable [Kambhampati and Nau, 1996]. However, any such POP can always be made POCL-valid by adding ordering constraints [Bercher and Olz, 2020].

\subsection{Partial Weighted MAXSAT}

The partial weighted maximum satisfiability (partial weighted MAXSAT) problem is a generalised optimisation variant of SAT that distinguishes between soft clauses, which are assigned a numeric weight, and hard clauses, which are unweighted. The aim of the partial weighted MAXSAT problem is to find an interpretation that satisfies all hard clauses and maximises the total weight of the satisfied soft clauses. The $k$ syntax $\phi$ indicates that clause $\phi$ has weight $k$, and no weight marking indicates a hard clause. For example, the formula $\left(p_{1} \vee p_{2}\right) \wedge \neg p_{1} \wedge \neg p_{2}$ is trivially unsatisfiable, but the interpretation $\left\{p_{1} / \top, p_{2} / \perp\right\}$ is an optimal solution to the partial weighted MAXSAT problem.

\section{Optimality Criteria for Partial-Order Plans}

Bäckström [1998]'s seminal work on action ordering and plan flexibility considers two types of modification: deordering, in which constraints can be removed but not added, and reordering, which allows both. Both must result in a valid POP:

Definition 8. Let $P=\langle O, \theta, \prec\rangle$ and $Q=\left\langle O, \theta, \prec^{\prime}\right\rangle$ be two POPs. Then:

- $Q$ is a reordering of $P$ iff they are both valid.

- $Q$ is a deordering of $P$ iff $i t$ is a reordering and $\prec^{\prime} \subseteq \prec$.

- $Q$ is a strict deordering of $P$ iff it is a deordering and $\prec^{\prime} \subset \prec$.

Bäckström defines the relative optimality of two POPs by comparing their ordering constraints. A minimal deordering of a POP cannot be relaxed any further without rendering the POP invalid, and a minimum deordering is the smallest of all valid deorderings of a POP. A minimum reordering has the fewest possible ordering constraints while remaining valid:

Definition 9. Let $P=\langle O, \theta, \prec\rangle$ and $Q=\left\langle O, \theta, \prec^{\prime}\right\rangle$ be two POPs. Then:

- $Q$ is a minimal deordering of $P$ iff $Q$ is a deordering of $P$ and there is no POP $R$ such that $R$ is a strict deordering of $Q$.

- $Q$ is a minimum deordering of $P$ iff $Q$ is a deordering of $P$ and there is no POP $R=\left\langle O, \theta, \prec^{\prime \prime}\right\rangle$ such that $R$ is a deordering of $P$ and $\left|\prec^{\prime \prime}\right|<\left|\prec^{\prime}\right|$.

- $Q$ is a minimum reordering of $P$ iff $Q$ is a reordering of $P$ and there is no POP $R=\left\langle O, \theta, \prec^{\prime \prime}\right\rangle$ such that $R$ is a reordering of $P$ and $\left|\prec^{\prime \prime}\right|<\left|\prec^{\prime}\right|$.
While a minimal deordering of a given POP can be found in polynomial time, deciding whether there exists a de/reordering with fewer than $k$ ordering constraints is NPcomplete, and finding a minimum de/reordering is NP-hard and cannot be approximated within a constant factor.

A key limitation of the optimality criteria above is the assumption that the POP's variable bindings remain static. We thus define more generalised criteria that accommodate modifications to a POP's variable bindings. In all cases, the resulting POP must remain both ground (i.e., its variable bindings must be complete and ground w.r.t. $O$ ) and valid:

Definition 10. Let $P=\langle O, \theta, \prec\rangle$ and $Q=\left\langle O, \theta^{\prime}, \prec^{\prime}\right\rangle$ be two POPs. Then:

- $Q$ is a reinstantiated reordering of $P$ iff both $P$ and $Q$ are valid.

- $Q$ is a reinstantiated deordering of $P$ iff $Q$ is a reinstantiated reordering of $P$ and $\prec^{\prime} \subseteq \prec$.

- $Q$ is a reinstantiated strict deordering of $P$ iff $Q$ is a reinstantiated deordering of $P$ and $\prec^{\prime} \subset \prec$.

For example, plans $P_{1}-P_{3}$ in Figure 1 use the same operators, and so they are reinstantiated reorderings of each other, whereas $P_{3}$ is a reinstantiated strict deordering of $P_{1}$ and $P_{2}$.

The notion of a "least-constrained" POP can now be generalised to allow for changes in variable bindings. A minimal reinstantiated deordering of a $\mathrm{POP}$ is a reinstantiated deordering that cannot be relaxed any further: no modification to the POP's variable bindings will allow for the removal of any ordering constraints. A minimum reinstantiated deordering of a POP has the fewest ordering constraints of all of its reinstantiated deorderings. Of all the possible modifications to a POP's ordering and binding, a minimum reinstantiated reordering contains the fewest ordering constraints:

Definition 11. Let $P=\langle O, \theta, \prec\rangle$ and $Q=\left\langle O, \theta^{\prime}, \prec^{\prime}\right\rangle$ be two POPs. Then:

- $Q$ is a minimal reinstantiated deordering of $P$ iff $Q$ is a reinstantiated deordering of $P$ and there is no POP $R$ such that $R$ is a reinstantiated strict deordering of $Q$.

- $Q$ is a minimum reinstantiated deordering of $P$ iff $Q$ is a reinstantiated deordering of $P$ and there is no POP $R=\left\langle O, \theta^{\prime \prime}, \prec^{\prime \prime}\right\rangle$ such that $R$ is a reinstantiated deordering of $P$ and $\left|\prec^{\prime \prime}\right|<\left|\prec^{\prime}\right|$.

- $Q$ is a minimum reinstantiated reordering of $P$ iff $Q$ is a reinstantiated reordering of $P$ and there is no $P O P$ $R=\left\langle O, \theta^{\prime \prime}, \prec^{\prime \prime}\right\rangle$ such that $R$ is a reinstantiated reordering of $P$ and $\left|\prec^{\prime \prime}\right|<\left|\prec^{\prime}\right|$.

For example, plan $P_{3}$ has two ordering constraints, and as there is no reinstantiation of $P_{1}$ which allows for fewer than this, $P_{3}$ is a minimum reinstantiated reordering of $P_{1}$.

\section{Complexity Results}

Deciding whether a POP has a reinstantiated de/reordering with fewer than $k$ ordering constraints is NP-complete, and the optimisation problem of finding a minimum reinstantiated de/reordering cannot be approximated within a constant factor (proofs for minimum reinstantiated deordering are provided, those for reordering are a trivial modification): 
Theorem 1. Given a POP $P=\langle O, \theta, \prec\rangle$ and an integer $k>$ 0 , determining whether there exists a POP $Q=\left\langle O, \theta^{\prime}, \prec^{\prime}\right\rangle$ s.t. $Q$ is a reinstantiated deordering (or reordering) of $P$ and $\left|\prec^{\prime}\right|<k$ is NP-complete.

Proof. For membership, guess a POP $Q=\left\langle O, \theta^{\prime}, \prec^{\prime}\right\rangle$ and verify in P-time that $Q$ is valid, $\prec^{\prime} \subseteq \prec$ and $\left|\prec^{\prime}\right|<k$. Hardness is by reduction from the NP-complete [Bäckström, 1998] decision problem of minimum deordering, which asks whether $P$ has a deorder with $<k$ ordering constraints. Construct $P^{\prime}=\left\langle O^{\prime}, \theta, \prec\right\rangle$ where $o \in O$ iff there exists a $o^{\prime} \in O^{\prime}$ s.t. $\operatorname{vars}\left(o^{\prime}\right)=\operatorname{vars}(o)$, post $\left(o^{\prime}\right)=\operatorname{post}(o)$ and $\operatorname{pre}\left(o^{\prime}\right)=\operatorname{pre}(o) \cup\left\{x=\theta(x): x \in \operatorname{vars}\left(o^{\prime}\right)\right\}$. As the operators in $O^{\prime}$ have preconditions that "fix" the variable bindings, $P$ has a deorder with $<k$ ordering constraints iff $P^{\prime}$ has a reinstantiated deorder with $<k$ ordering constraints.

Theorem 2. The problem of finding a minimum reinstantiated deordering (or reordering) of a POP is not in APX unless $\mathrm{NP} \subseteq \mathrm{DTIME}\left(n^{\text {poly } \log n}\right)$.

Proof. Proof is by reductio. Assume that minimum reinstantiated deordering is in APX. Then there must be a function, $A$, that approximates it within a constant factor. As the reduction in the Theorem 1 proof preserves solutions, $A$ also approximates minimum deordering within a constant factor, which is impossible unless NP $\subseteq$ $\operatorname{DTIME}\left(n^{\text {poly } \log \mathrm{n}}\right)$ [Bäckström, 1998].

\section{Optimality Under POCL-validity}

Under the additional requirement that the input and optimised POPs be POCL-valid, minimum de/reordering remain NPcomplete (a trivial corollary of Theorem 4.8 in [Bäckström, 1998]). However, due to the stronger requirements of POCLvalidity there exist minimum POCL-valid de/reorderings that can be further optimised while remaining valid under Definition 3 [Kambhampati and Nau, 1996]. These complexity and completeness results can be trivially extended to minimum reinstantiated POCL-valid de/reordering.

\section{Partial Weighted MaxSAT Encoding}

The problem of optimising a POP's ordering can be naturally expressed as an instance of the partial weighted MAXSAT problem (Section 2.3). This approach was introduced by Muise et al. [2016], whose MD and MR encodings transform a POP into MAXSAT instances with optimal solutions corresponding to minimum POCL-valid de/reorderings, resp.

This section introduces MRD and MRR: generalised encodings that allow the POP to be reinstantiated. Their optimal solutions therefore correspond to minimum POCL-valid reinstantiated de/reorderings, resp. They also optimise MD and MR by breaking symmetries and removing propositions and clauses that are not required to enforce plan validity.

We note due to the difficulty of expressing Definition 3 into MAXSAT, all four encodings instead preserve plan validity with the POCL requirements in Definition 7. While easier to encode, this raises the possiblity that the resulting optimised POPs contain unnecessary ordering constraints.

\subsection{The MD and MR Encodings}

MD and MR use two "types" of propositional variables. If $P=\langle O, \prec, \theta\rangle$ is the input POP, then for each pair of actions $a_{1}, a_{2} \in O \theta$, a proposition $p_{a_{1} \prec a_{2}}$ is introduced to indicate that $a_{1}$ must precede $a_{2}$ in the resulting POP. For all $a_{c}, a_{p}$ and $q$ such that $a_{c}$ consumes $q$ and $a_{p}$ produces $q$, a proposition $p_{a_{p}, q, a_{c}}$ is introduced to encode the requirement that in the final POP, $a_{p}$ be causally linked to $a_{c}$ with respect to $q$.

Formulae 1-3 ensure that the output POP is acyclic and transitively closed with all actions ordered between the initial state and goal:

$$
\begin{aligned}
& \bigwedge_{a} \neg p_{a \prec a} . \\
& \bigwedge_{a_{1}, a_{2}, a_{3}} p_{a_{1} \prec a_{2}} \wedge p_{a_{2} \prec a_{3}} \rightarrow p_{a_{1} \prec a_{3} .} . \\
& \bigwedge_{\notin\left\{a_{I}, a_{G}\right\}} p_{a_{I} \prec a} \wedge p_{a \prec a_{G}} .
\end{aligned}
$$

Formulae 4 and 5 encode POCL-validity as in Definition 7:

$$
\begin{aligned}
& \bigwedge_{a_{p}, a_{c}, q:}\left(p_{a_{p}, q, a_{c}} \rightarrow \bigwedge_{a_{t}: a_{t} \neq a_{a_{c}} \prec a_{p}} \vee p_{a_{c} \prec a_{t}}\right) . \\
& \begin{array}{cc}
a_{t}: a_{t} \neq a_{c}, \\
\operatorname{cons}\left(a_{c}, q\right), & \operatorname{thrtns}\left(a_{t}, q\right)
\end{array} \\
& \left.\operatorname{prods}\left(a_{p}, q\right)\right) \\
& \bigwedge_{\substack{a_{c}, q: \\
\operatorname{cons}\left(a_{c}, q\right)}} \bigvee_{a_{p}: \operatorname{prods}\left(a_{p}, q\right)} p_{a_{p} \prec a_{c}} \wedge p_{a_{p}, q, a_{c}} .
\end{aligned}
$$

Formula 6 adds a soft unit clause with the negation of each ordering proposition, meaning that higher weight solutions will have fewer ordering constraints:

$$
\bigwedge_{a_{1}, a_{2}} \neg p_{a_{1} \prec a_{2}}^{1}
$$

Definition 12. MR encodes a POP into a partial weighted MAXSAT instance through Formulae 1-6.

The MD encoding extends MR with clauses that disallow any ordering constraints that were not in the input plan, thus forcing the resulting POP to be deordering of the input:

$$
\bigwedge_{\left(a_{1}, a_{2}\right) \notin \prec} \neg p_{a_{1} \prec a_{2}} .
$$

Definition 13. MD encodes a POP into a partial weighted MAXSAT instance through Formulae 1-7.

\subsection{The MRD and MRR Encodings}

MRD and MRR allow the input POP to be reinstantiated, and so use different variable "types" to express the allowable rebindings. Three types are used, as per the following sets:

- $\mathcal{P}_{\prec}$ contains propositions of the form $p_{O_{1} \prec o_{2}}$, which indicate that $o_{1} \prec o_{2}$ in the resulting POP.

- $\mathcal{P}_{\theta}$ contains propositions of the form $p_{t=u}$, which encode a requirement that in the final $\operatorname{POP} \theta(t)=\theta(u)$. 


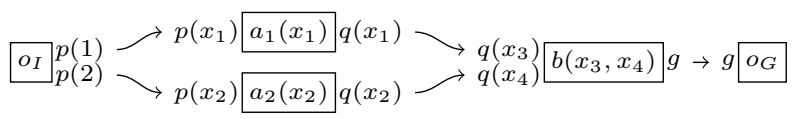

Figure 2: Plan $P_{4}$ from a synthetic domain. Pre/postconditions are to the left/right of operators, resp., and arrows indicate causal links.

- $\mathcal{P}_{C}$ contains propositions of the form $p_{o_{p}, \vec{t}, o_{c}, \vec{u}}$, indicating that there must be some $q$ s.t. causal link $\left\langle o_{p}, q(\vec{t}), o_{c}, q(\vec{u})\right\rangle$ is unthreatened in the final POP.

Muise et al. [2016] observed that the cubic number of clauses generated by Formula 2 make MD and MR infeasable for large plans. As this is exacerbated in MRD and MRR by the need to also close the variable equality relation, a number of optimisations are introduced.

The first removes any ordering or binding constraints (and propositions that encode them) that are not required to preserve causal links. For example, consider Plan $P_{4}$ in Figure 2. Operator $a_{1}$ cannot be used to produce any of $a_{2}$ 's preconditions, and nor does it threaten any of its postconditions (nor vice-versa). Therefore, no minimum de/reordering of $P_{4}$ will require that $a_{1} \prec a_{2}, a_{2} \prec a_{1}$ or that $x_{1}$ and $x_{2}$ be (non-) codesignated. MRD and MRR therefore exclude the propositions $p_{a_{1} \prec a_{2}}, p_{a_{2} \prec a_{1}}, p_{x_{1}=x_{2}}$ and $p_{x_{2}=x_{1}}$.

The second optimisation prevents the encoding of possible causal links if the required ordering constraints are known to be unsatisfiable. For example, Formula 7 in MD enforces deordering with negated unit clauses. Rather than include propositions that are a priori false, MRD removes the proposition and limits the possible causal links to those where the consumer did not precede the producer in the input POP. Additionally, POPs can contain multiple operators of the same type (e.g., the two instances of $a$ in plan $P_{4}$ ), resulting in an exponential number of de/reorderings that are equivalent under permutation of the operators. As is standard [Say et al., 2016; Shlyakhter, 2007], we (partially) break these symmetries by limiting ordering constraints between operators of the same type to those found in an arbitrary linearisation. However, rather than achieve this with additional symmetry breaking clauses, we limit $\mathcal{P}_{\prec}$ and $\mathcal{P}_{C}$ as above.

The definition below formalises and generalises these ideas. If $o_{1}$ and $o_{2}$ are operators and $\prec_{A}$ is a set of "allowable" orderings, then $o_{1}$ and $o_{2}$ are causally related iff $o_{1} \prec_{A} O_{2}$ and there is a possible causal or threat relationship between them:

Definition 14. $o_{1}, o_{2}, q(\vec{t})$ and $q(\vec{u})$ are causally related w.r.t. $\prec_{A}$, iff $o_{1} \prec_{A} O_{2}$ and either:

1. $\operatorname{prods}\left(o_{1}, q(\vec{t})\right)$ and cons $\left(o_{2}, q(\vec{u})\right)$,

2. $\operatorname{thrtns}\left(o_{1}, q(\vec{t})\right)$ and $\operatorname{prods}\left(o_{2}, q(\vec{u})\right)$, or

3. $\operatorname{cons}\left(o_{1}, q(\vec{t})\right)$ and thrtns $\left(o_{2}, q(\vec{u})\right)$.

The sets of propositional variables can now be defined:

Definition 15. Let $P=\langle O, \theta, \prec\rangle$ be a $P O P$ and $\prec_{A} \subseteq O^{2}$ be a set of allowable orderings. Then $\mathcal{P}_{\prec}, \mathcal{P}_{\theta}$, and $\mathcal{P}_{C}$ are the smallest sets s.t.:

- $p_{o_{1} \prec o_{2}} \in \mathcal{P}_{\prec}$ iff either (i) there exists a $q(\vec{t})$ and $q(\vec{u})$ s.t. $o_{1}, o_{2}, q(\vec{t})$ and $q(\vec{u})$ are causally related w.r.t. $\prec_{A}$, or (ii) there exists an $o_{3}$ s.t. $p_{o_{1} \prec o_{3}}, p_{O_{3} \prec o_{2}} \in \mathcal{P}_{\prec}$.
- $p_{t=u} \in \mathcal{P}_{\theta}$ iff either (i) there exists a $o_{1}, o_{2}, q(\vec{t})$ and $q(\vec{u})$ that are causally related w.r.t. $\prec_{A}$ and some $i$ s.t. $\vec{t}[i]=t, \vec{u}[i]=u$, or (ii) there exists a $v$ s.t. $p_{t=v}, p_{v=u} \in$ $\mathcal{P}_{\theta}{ }^{1}$

- $p_{o_{p}, \vec{t}, o_{c}, \vec{u}} \in \mathcal{P}_{C}$ iff there exists a $q$ s.t. $\operatorname{prods}\left(o_{p}, q(\vec{t})\right)$, $\operatorname{cons}\left(o_{c}, q(\vec{u})\right)$ and $o_{p} \prec_{A} o_{c}$.

MRD and MRR encode a POP $P=\langle O, \theta, \prec\rangle$ and a set of allowable orderings $\prec_{A} \subseteq O^{2}$ through the following sets of clauses. Formulae 8-10 ensure that any solution represents an acyclic, transitively closed POP where all operators precede the goal and are preceded by the initial state. They derive from Formulae 1-3 in MR, but have removed any propositions and constraints not required to preserve validity:

$$
\begin{aligned}
& \bigwedge \neg p_{o_{1} \prec o_{2}} \vee \neg p_{o_{2} \prec o_{1}} . \\
& p_{o_{1} \prec o_{2}}, p_{o_{2} \prec o_{1}} \in \mathcal{P} \prec \\
& \bigwedge p_{o_{1} \prec o_{2}} \wedge p_{O_{2} \prec o_{3}} \rightarrow p_{o_{1} \prec o_{3}} . \\
& \begin{array}{c}
p_{o_{1} \prec o_{2}}, p_{o_{1} \prec o_{3}}, \\
p_{o_{2} \prec o_{3}} \in \mathcal{P} \prec
\end{array} \\
& \bigwedge p_{O_{I} \prec o} \wedge p_{o \prec o_{G}} . \\
& p_{o_{I} \prec o, p_{o \prec o} \in \mathcal{P} \prec}
\end{aligned}
$$

Formulae 11 and 12 ensures that the equality relation over variables and constants is symmetric and transitive, and Formula 13 ensures that each variable is bound to exactly one object. They are extensions to MD and MR that ensure the consistency of the POP's variable bindings:

$$
\begin{aligned}
& \bigwedge p_{t=u} \leftrightarrow p_{u=t} \\
& p_{t=u} \in \mathcal{P}_{\theta} \\
& \bigwedge p_{t=u} \wedge p_{u=v} \rightarrow p_{t=v} . \\
& \begin{array}{c}
p_{t=u}, p_{t=v}, \\
p_{u=v} \in \overline{\mathcal{P}}_{\theta}
\end{array} \\
& \bigwedge \quad\left(\bigvee p_{x=c} \wedge \bigwedge \neg p_{x=c_{1}} \vee \neg p_{x=c_{2}}\right) \\
& x \in \operatorname{vars}(O) \quad c \in \text { consts }(O): \quad c_{1}, c_{2} \in \operatorname{consts}(O) \text { : } \\
& \begin{array}{c}
p_{x=c} \in \mathcal{P}_{\theta} \quad p_{x=c_{1}}, p_{x=c_{2}} \in \mathcal{P}_{\theta}, \\
c_{1} \neq c_{2}
\end{array}
\end{aligned}
$$

Formulae 14 and 15 encode POCL-validity. Formula 14 requires that each consumer be causally linked to at least one producer, and Formula 15 defines the ordering, binding and threat protection constraints required for a causal link to hold. These derive from Formulae 4 and 5 in MD and MR, but have been optimised to remove redundant propositions, and generalised to allow the POP's variable bindings to change:

$$
\begin{aligned}
& \bigwedge \quad \bigvee p_{o_{p}, \vec{t}, o_{c}, \vec{u}} \\
& \begin{array}{c}
o_{c}, q(\vec{u}): \\
\operatorname{cons}\left(o_{c}, q(\vec{u})\right)
\end{array} \quad p_{o_{p}, \vec{t}, o_{c}, \vec{u}} \in \mathcal{P}_{C} \\
& \bigwedge p_{o_{p}, \vec{t}, o_{c}, \vec{u}} \rightarrow\left[\bigwedge p_{\vec{t}[i]=\vec{u}[i]} \wedge p_{o_{p} \prec o_{c}} \wedge\right. \\
& p_{o_{p}, \vec{t}, o_{c}, \vec{u}} \in \mathcal{P}_{C} \quad 1 \leq i \leq|\vec{t}| \\
& \bigwedge_{\substack{o_{t}, q(\vec{v}): o_{t} \neq o_{c}, \operatorname{thrtns}\left(o_{t}, q(\vec{v})\right)}}^{\left(\bigvee_{p \in \mathcal{P}_{\prec} \cap}\right.} \underset{\substack{\left\{p_{o_{t} \prec o_{p}}, p_{o_{c} \prec o_{t}}\right\} \\
\left\{p_{\vec{t}[i]=\vec{v}[i]}: 1 \leq i \leq|\vec{t}|\right\}}}{\left.\left.\bigvee_{p \in \mathcal{P}_{\theta} \cap} \neg p\right)\right] .}
\end{aligned}
$$

\footnotetext{
${ }^{1}$ Implemented code merges all $p_{t=u}$ and $p_{u=t}$ and updates Formulae 11 and 12 accordingly.
} 
Formula 16, an optimised version of Formula 6 in MD and MR, adds soft clauses that minimise the ordering constraints:

$$
\bigwedge_{p_{o_{1} \prec o_{2} \in \mathcal{P}} \neg p_{o_{1} \prec o_{2}} .}^{1} .
$$

The MRD and MRR encodings can now be defined. The allowed orderings for MRD are those from the input plan, forcing the resulting POP to be a deordering of the input:

Definition 16. MRD encodes a POP $P=\langle O, \theta, \prec\rangle$ into a partial weighted MAXSAT instance through Formulae 8-16, with the allowable orderings $\prec_{A}=\prec$.

The MRR encoding only disallows orderings for the purposes of symmetry breaking:

Definition 17. If $P=\langle O, \theta, \prec\rangle$ is a $P O P$ and $\prec^{\prime}$ is an arbitrary linearisation of $P$, then MRR encodes $P$ into a partialweighted MAXSAT instance through Formulae 8-16, with the allowable orderings $\prec_{A}=O^{2} \backslash\left\{\left(o_{2}, o_{1}\right)\right.$ : name $\left(o_{1}\right)=$ name $\left.\left.\left(o_{2}\right), o_{1} \prec^{\prime} o_{2}\right\}\right)$.

\section{Experimental Evaluation}

While a minimum reinstantiated de/reorder of a POP can never be less flexible than a minimum de/reorder (Definitions 9 and 11), the question remains whether, in practice, reinstantiation provides any significant increase in flexibility. The search for a minimum de/reorder often times out before an optimal (or indeed any) solution has been found [Muise et al., 2016], and allowing variable bindings creates a harder problem with an exponentially larger search space. This evaluation will thus address the question of whether reinstantiated de/reordering can provide more flexibility than standard de/reordering under the same resource constraints.

\subsection{Experimental Setup}

We answer the question above by comparing optimised plans produced by the Loandra MAXSAT solver [Berg et al., 2019] using the MD, MR, MRD and MRR encodings.

We also compare the MAXSAT-based techniques with the explanation-based order generalisation (EOG) deordering technique of Kambhampati and Kedar [1994]. First, a validation structure is constructed: a subset of $L_{P}$ (Definition 6) that links each consumer to its earliest producer:

Definition 18. If $P=\langle O, \theta, \prec\rangle$ is a POP then $V_{P}$ is a validation stucture of $P$ iff $V_{P} \subseteq L_{P}$ and there exists a total order $\prec \subseteq \prec^{\prime}$ s.t. if $\left\langle o_{p}, q(\overrightarrow{\vec{t}}), o_{c}, q(\vec{u})\right\rangle \in V_{P}$ and $\left\langle o_{p}^{\prime}, q\left(\vec{t}^{\prime}\right), o_{c}, q(\vec{u})\right\rangle \in L_{P}$ then $o_{p} \prec^{\prime} o_{p}^{\prime}$.

This validation structure serves as a deordering heuristic:

Definition 19. If $P$ is a POP with validation structure $V_{P}$, then the explanation-based order generalisation of $P$ w.r.t. $V_{P}$ is the POP $P^{\prime}=\left\langle O, \theta, \prec^{\prime+}\right\rangle$ where $o_{1} \prec^{\prime} o_{2}$ iff there exists a $q(\vec{t}), q(\vec{u})$ s.t. either:

1. $\left\langle o_{1}, q(\vec{t}), o_{2}, q(\vec{u})\right\rangle \in V_{P}$,

2. there exists an $o_{3}$ and $q(\vec{v})$ s.t. $\left\langle o_{2}, q(\vec{u}), o_{3}, q(\vec{v})\right\rangle \in V_{P}$, $o_{1} \prec o_{2}$ and $\operatorname{thrtns}\left(o_{1}, q(\vec{u})\right)$, or

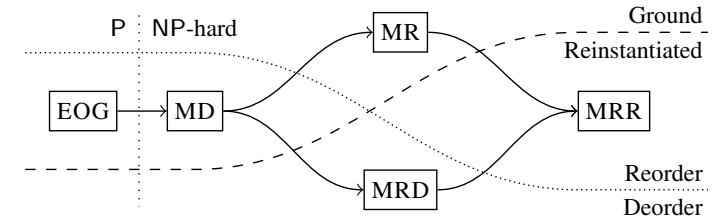

Figure 3: POP optimisation hierarchy. Arrows lead from less to more general optimisations.

3. there exists an $o_{3}$ and $q(\vec{v})$ s.t. $\left\langle o_{3}, q(\vec{v}), o_{1}, q(\vec{u})\right\rangle \in V_{P}$, $o_{1} \prec o_{2}$ and thrtns $\left(o_{2}, q(\vec{u})\right)$.

While EOG cannot guarantee even a minimal deorder of its input [Bäckström, 1998], it can be computed in polynomial time and in practice consistently finds minimum deorderings [Muise et al., 2016]. It thus serves an effective baseline for evaluating the MAXSAT-based optimisation techniques.

Figure 3 depicts the hierarchy of (the problems solved by) these algorithms. EOG is in $\mathrm{P}$, all others are NP-hard to compute optimally. MD (minimum deorder) searches for a minimally ordered POP but disallows reorderings and changes to variable bindings. This is relaxed by both MR (minimum reorder), which allows reorderings, and MRD (minimum reinstantiated deorder), which allows bindings to change. The most general is MRR (minimum reinstantiated reorder), which allows both reorderings and changes to bindings.

Test cases (i.e., input plans) were generated by giving all first-order IPC STRIPS planning instances to three planners. To ensure a variety of plans, three planners of distinct "types" were used: the novelty-driven best-first search planner DualBFWS [Lipovetzky and Geffner, 2017], the heuristic forwardsearch planner LAMA [Richter and Westphal, 2010] and the SAT planner Madagascar [Rintanen, 2010]. Each (unique) plan was encoded with each encoding ${ }^{2}$, and the resulting MAXSAT instances were preprocessed with MaxPre [Korhonen et al., 2017] and given to the Loandra MAXSAT solver. Plan generation and encoding/optimisation were both limited to $8 \mathrm{~GB}$ and $30 \mathrm{~m}$ at $2.60 \mathrm{GHz}$.

Because the optimality criteria in Definitions 9 and 11 cannot compare POPs with different operators, POP flexibilty is here assessed with the commonly-used flex measure [Nguyen and Kambhampati, 2001; Muise et al., 2016; Siddiqui and Haslum, 2012], which is computed from the proportion of operators that are not (transitively) ordered:

Definition 20. If $P=\langle O, \theta, \prec\rangle$ is a POP then $\operatorname{flex}(P) \stackrel{\text { def }}{=}$ $1-\left(|\prec| / \sum\{i: 1 \leq i<|O|\}\right)$.

A POP's flex ranges from 0 to 1 (a totally-ordered and unordered POP, resp.) and is a strong indicator of the number of linearisations it represents [Muise et al., 2016].

\subsection{Results}

Results are summarised in Table 1. Domains where no plan was improved by any technique (agricola, pegsol, snake, organic-synth-split, sokoban, visitall) have been excluded.

\footnotetext{
${ }^{2}$ Implementation is available at bitbucket.org/max_waters/mrr
} 


\begin{tabular}{|c|c|c|c|c|c|c|c|c|c|c|}
\hline \multirow[b]{2}{*}{ Domain } & \multirow[b]{2}{*}{$f_{\mathrm{EOG}}$} & \multicolumn{3}{|c|}{ MR } & \multicolumn{3}{|c|}{ MRD } & \multicolumn{3}{|c|}{ MRR } \\
\hline & & $T$ & $C$ & $\Delta_{\mathrm{EOG}}$ & $T$ & $C$ & $\Delta_{\text {EOG }}$ & $T$ & $C$ & $\Delta_{\mathrm{EOC}}$ \\
\hline airport (115) & 0.25 & 0.13 & $63 \%$ & $30 \%$ & 1.28 & $28 \%$ & $0 \%$ & 1.9 & $24 \%$ & $30 \%$ \\
\hline barman (74) & 0.01 & 30 & $41 \%$ & & 2.75 & $77 \%$ & & 28.79 & $36 \%$ & \\
\hline childsnack (90) & 0.69 & 15.77 & $100 \%$ & $4 \%$ & 20.64 & $100 \%$ & $6 \%$ & 29.99 & $99 \%$ & $6 \%$ \\
\hline data $(22)$ & 0.36 & 6.93 & $86 \%$ & & 25.53 & $50 \%$ & & 30 & $14 \%$ & \\
\hline depot (63) & 0.38 & 9.85 & $90 \%$ & $8 \%$ & 15.02 & $71 \%$ & $28 \%$ & 26.42 & $44 \%$ & $44 \%$ \\
\hline driverlog (59) & 0.37 & 4 & $97 \%$ & $2 \%$ & 7.35 & $78 \%$ & $17 \%$ & 15.9 & $73 \%$ & $34 \%$ \\
\hline elevators (101) & 0.43 & 3.71 & $65 \%$ & & 6.28 & $59 \%$ & $12 \%$ & 24.09 & $56 \%$ & $28 \%$ \\
\hline floortile (65) & 0.39 & 20.2 & $100 \%$ & & 16.58 & $98 \%$ & & 30 & $5 \%$ & \\
\hline freecell (147) & 0.14 & 1.08 & $100 \%$ & $2 \%$ & 7.07 & $95 \%$ & $40 \%$ & 21.59 & $56 \%$ & $\mathbf{9 4} \%$ \\
\hline $\operatorname{ged}(91)$ & 0.11 & 9.6 & $92 \%$ & & 9.7 & $65 \%$ & $0 \%$ & 10.21 & $60 \%$ & \\
\hline gripper (60) & 0.02 & 26.33 & $85 \%$ & $19 \%$ & 0.39 & $100 \%$ & $0 \%$ & 25.15 & $42 \%$ & $29 \%$ \\
\hline hiking (82) & 0.13 & 4.03 & $94 \%$ & $6 \%$ & 8.08 & $96 \%$ & $22 \%$ & 20.96 & $44 \%$ & $42 \%$ \\
\hline logistics98 (101) & 0.64 & 5.34 & $67 \%$ & $3 \%$ & 20.55 & $45 \%$ & $8 \%$ & 26.92 & $28 \%$ & $9 \%$ \\
\hline mprime (97) & 0.22 & 0.15 & $100 \%$ & & 13.11 & $77 \%$ & $54 \%$ & 14.1 & $78 \%$ & $60 \%$ \\
\hline mystery (51) & 0.22 & 0.06 & $100 \%$ & & 4.92 & $96 \%$ & $44 \%$ & 8.12 & $94 \%$ & $48 \%$ \\
\hline nomystery (95) & 0.04 & 2.72 & $100 \%$ & $12 \%$ & 9.22 & $96 \%$ & & 17.88 & $60 \%$ & $39 \%$ \\
\hline parcprinter (118) & 0.7 & 0.12 & $100 \%$ & $0 \%$ & 14.63 & $100 \%$ & & 24.95 & $99 \%$ & \\
\hline parking (80) & 0 & 0.86 & $100 \%$ & $0 \%$ & 26.73 & $35 \%$ & & 29.32 & $5 \%$ & \\
\hline pipesworld (89) & 0.19 & 0.85 & $100 \%$ & $2 \%$ & 7.42 & $91 \%$ & $74 \%$ & 21.99 & $62 \%$ & $\mathbf{9 6} \%$ \\
\hline rovers $(120)$ & 0.67 & 9.82 & $83 \%$ & $1 \%$ & 16 & $61 \%$ & $6 \%$ & 23.8 & $49 \%$ & $12 \%$ \\
\hline satellite (100) & 0.5 & 0.94 & $88 \%$ & & 21.39 & $66 \%$ & $4 \%$ & 25.76 & $40 \%$ & $8 \%$ \\
\hline scanalyzer (104) & 0.37 & 6.53 & $97 \%$ & $8 \%$ & 20.67 & $80 \%$ & $35 \%$ & 24.97 & $53 \%$ & $38 \%$ \\
\hline termes (22) & & 30 & $5 \%$ & & 8.44 & $27 \%$ & & 30 & $0 \%$ & \\
\hline tetris (79) & 0.63 & 0.78 & $96 \%$ & & 20.67 & $49 \%$ & $6 \%$ & 25.16 & $32 \%$ & $9 \%$ \\
\hline thoughtful (40) & 0.14 & 5.54 & $100 \%$ & & 12.21 & $100 \%$ & & 22.66 & $33 \%$ & \\
\hline tpp (89) & 0.34 & 13.66 & $66 \%$ & $13 \%$ & 19.46 & $52 \%$ & $32 \%$ & 23.11 & $43 \%$ & $49 \%$ \\
\hline transport (98) & 0.34 & 8.44 & $70 \%$ & & 18.21 & $58 \%$ & $37 \%$ & 26.84 & $=46 \%$ & $46 \%$ \\
\hline w-working (120) & 0.88 & 0.1 & $100 \%$ & $0 \%$ & 16.65 & $86 \%$ & $3 \%$ & 20.92 & $73 \%$ & $3 \%$ \\
\hline zenotravel (57) & 0.41 & 2.03 & $95 \%$ & $1 \%$ & 11.63 & $77 \%$ & $17 \%$ & 20.54 & $=65 \%$ & $21 \%$ \\
\hline$A L L(2429)$ & 0.4 & 6.06 & $87 \%$ & $2 \%$ & 13.52 & $74 \%$ & $13 \%$ & 22.43 & $52 \%$ & $20 \%$ \\
\hline
\end{tabular}

Table 1: For each domain, $f_{\mathrm{EOG}}$ is the mean flex produced by EOG, and for each encoder, $C$ is the $\%$ of plans for which a (satisficing or optimal) solution was found, $T$ is the mean run time in minutes, and $\Delta_{\mathrm{EOG}}$ is the mean $\%$ flex increase over EOG. All flex values are computed from plans for which every encoder found a solution, and all $\Delta_{\text {EOG }}$ values are statistically significant with $p<0.01$ as calculated from a single-tailed, paired t-test. Empty cells indicate no data, or no significant difference.

\section{Effectiveness of EOG}

Our results confirm those of Muise et al. [2016] that EOG always finds a minimum deorder of the input plan, despite a lack of optimality guarantees. Also, EOG takes $<3 s$ in $88 \%$ of plans, with nearly $100 \%$ coverage. We thus exclude MD from the rest of the discussion.

As well as consistently finding minimum deorders, EOG found a minimum reorder in $69 \%$ of plans the plans that were solved optimally by MR, a minimum reinstantiated deorder in $53 \%$ of the plans solved optimally by MRD, and a minimum reinstantiated reorder in $45 \%$ of the plans solved optimally by MRR. As EOG can neither reorder actions nor rebind variables, this means that in a significant number of plans, no reordering or reinstantiation was necessary in order to find an optimally ordered POP.

\section{Benefit of Reinstantiation}

To assess the practical benefit of reinstantiation, we compare the coverage, run time and final flex of MRD and MRR with EOG and MR. To allow a meaningful comparison, mean flex values are computed from plans for which a solution was found by all encoders.

Overall, MRR provides a $20 \%$ flex increase over EOG. ${ }^{3}$ This contrasts with the $3 \%$ flex increase provided by MR,

${ }^{3}$ All stated $f$ ex differences are statistically significant with $p<$ 0.01 as calculated from a single-tailed, paired t-test.

\begin{tabular}{|c|c|c|c|c|c|c|}
\hline \multirow[b]{2}{*}{ Encoding Size } & \multicolumn{2}{|c|}{ MR } & \multicolumn{2}{|c|}{ MRD } & \multicolumn{2}{|c|}{ MRR } \\
\hline & $n_{P}$ & C & $n_{P}$ & C & $n_{P}$ & C \\
\hline $0-10.0 \cdot 10^{4}$ & 1,197 & $100 \%$ & 358 & $100 \%$ & 268 & $100 \%$ \\
\hline $10.0 \cdot 10^{4}-2.0 \cdot 10^{5}$ & 210 & $100 \%$ & 221 & $100 \%$ & 180 & $99 \%$ \\
\hline $2.0 \cdot 10^{5}-4.0 \cdot 10^{5}$ & 181 & $100 \%$ & 241 & $100 \%$ & 213 & $94 \%$ \\
\hline $4.0 \cdot 10^{5}-8.0 \cdot 10^{5}$ & 154 & $99 \%$ & 265 & $95 \%$ & 248 & $85 \%$ \\
\hline $8.0 \cdot 10^{5}-1.6 \cdot 10^{6}$ & 167 & $99 \%$ & 250 & $89 \%$ & 259 & $68 \%$ \\
\hline $1.6 \cdot 10^{6}-3.2 \cdot 10^{6}$ & 112 & $85 \%$ & 269 & $82 \%$ & 241 & $44 \%$ \\
\hline $3.2 \cdot 10^{6}-6.4 \cdot 10^{6}$ & 82 & $30 \%$ & 202 & $57 \%$ & 220 & $27 \%$ \\
\hline $6.4 \cdot 10^{6}-1.3 \cdot 10^{7}$ & 33 & $15 \%$ & 211 & $50 \%$ & 229 & $14 \%$ \\
\hline $1.3 \cdot 10^{7}-2.6 \cdot 10^{7}$ & 6 & $0 \%$ & 119 & $38 \%$ & 206 & $15 \%$ \\
\hline $2.6 \cdot 10^{7}+$ & 1 & $0 \%$ & 7 & $14 \%$ & 79 & $0 \%$ \\
\hline
\end{tabular}

Table 2: Plan count $\left(n_{P}\right)$ and coverage $(C)$ by encoding size (in number of clauses) for MR, MRD and MRR. Note that the encoding size is a log scale, each size range being twice that of the previous.

meaning that reinstantiation has yielded a further flex increase of $17 \%$. This improvement is consistent: MRR times out before finding as flexible a solution as EOG in $<1 \%$ of the 1260 plans solved by both, with similar results for MR.

MRR improves on EOG in 24 domains. The largest differences are in freecell and pipesworld, where MRR provides flex increases of $94 \%$ and $96 \%$, resp., while MR provides $2 \%$, and mprime and transport, where MRR provides increases of $60 \%$ and $46 \%$, resp., and MR provides no significant benefit.

However, this additional flexibility comes at significant computational cost. MRR has a mean coverage and run time of $52 \%$ and $22.43 \mathrm{~m}$, resp., with coverage dropping significantly in floortile (5\%), parking (5\%) and termes (0\%).

The less general MRD approach provides less additional flex at less computational cost. With a mean coverage and run time of $74 \%$ and $13.52 \mathrm{~m}$, resp., it provides an overall flex increase of $13 \%$ over EOG and statistically significant increases in 21 domains, pipesworld being the highest (74\%).

Interestingly, the decrease in coverage is not simply due to larger MAXSAT formulae. Table 2 shows that, as expected, MRD and MRR generate the largest encodings, and coverage always decreases with encoding size. However, within each size range, the encoders' coverages differ significantly (e.g., MRD and MR always have more coverage than MRR), suggesting that the problem is the formulae's structure, not size. A likely cause is so-called object symmetries: combinations of differently labelled but functionally equivalent domain objects (e.g., rovers $R_{1}$ and $R_{2}$ in Figure 1). Their presence can result in a small increase in formula size but an exponential number of equivalent (non-) solutions.

\section{Influence of Planner}

Finally, we note that the source of the input plan influences plan flexibility. Madagascar produces POPs, more specifically parallel plans where each step is a set of unordered actions. Interestingly, EOG can still increase their initial mean flex of 0.08 to 0.5 by removing unnecesary ordering constraints produced by the division of the plan into steps. MRR further improves on this by $15 \%$. Over the sequential (flex = 0 ) plans generated by Dual-BFWS and LAMA, EOG gives a mean flex of 0.38 and 0.36 , resp., which is improved upon by MRR by $30 \%$ and $14 \%$, resp. Thus, while Dual-BFWS benefits most from reinstantiation, optimised Madagascar plans are the most flexible. 


\section{Discussion}

This paper presented a practical technique for plan optimisation through the modification of both ordering and variable binding constraints. Results show that in $52 \%$ of cases, MRR provides a flex increase of $20 \%$ over the EOG baseline in $22 \mathrm{~m}$, with results varying by domain. However, as reflected by the low coverage of MRR, this comes with considerable commputational cost. Additionally, in a significant number of cases, the additional computation simply reveals that the original bindings were already optimal.

Whether MRR is preferable to less costly methods such as EOG depends on the application. If offline preprocessing time is available and execution-time flexibilty is paramount, or the application domain is one where reinstantiation can quickly and consistently improve plan flexibility (e.g., mystery), then reinstantiation is clearly worthwhile.

This work generalises the POP optimality definitions of Bäckström [1998] and optimisation techniques of Muise $e t$ al. [2016] to allow changes in variable bindings. Other POP optimisation techniques have been studied. Muise $e t$ al. present extensions to MD and MR that also minimise the POP's size by removing any actions without dependent consumers (a problem shown to be NP-complete by $\mathrm{Olz}$ and Bercher [2019]), and Bercher and Olz [2020] study the theoretical aspects of modifying a POP's orderings in order to minimise its makespan. Siddiqui and Haslum [2012]'s block decomposition partitions a POP's actions into macrooperator blocks. The implicit requirement that blocks not be interleaved means that this approach can sometimes deorder plans that cannot be deordered by standard methods. Say et al. [2016] present MILP models for optimising POP size and flexibility. They avoid the cubic size of Formula 2 by using variables to represent action start and end times, resulting in significant efficiency gains over MD and MR.

Unlike the work presented here, the above techniques do not allow variable bindings to change. Thus, further work could investigate whether generalising these techniqes by allowing reinstantiation results in more flexible block decompositions or further reductions in plan size or makespan, and whether a MILP encoding of MRD and MRR can provide an improvement in execution time.

More general optimisation approaches relax a plan into a partial plan, which need not completely specify orderings or bindings. While a POP's linearisations are different orderings of the same set of actions, a partial plan defines of a set of classical plans that differ in both their ordering and variable bindings. Kambhampati and Kedar [1994] generalise EOG, and keep only the ordering and binding constraints needed to enforce POCL validity. Waters et al. [2018] show that partial plan constraints are intractable, limiting their execution-time use, and so present an algorithm that searches for partial plans that can be instantiated in polynomial time.

In Section 5, we suggest that object symmetries prevent effective reinstantiation. As there is much work on symmetry in planning [Joslin and Roy, 1997; Riddle et al., 2016; Fox and Long, 1999; Rintanen, 2003; Pochter et al., 2011], symmetry breaking in the context of plan optimisation is an interesting area for future work.

\section{References}

[Aghighi and Bäckström, 2017] Meysam Aghighi and Christer Bäckström. Plan reordering and parallel execution - A parameterized complexity view. In Proc. of AAAI, pages 3540-3546, 2017.

[Bäckström, 1998] Christer Bäckström. Computational aspects of reordering plans. Journal of Artificial Intelligence Research, 9:99-137, 1998.

[Bercher and Olz, 2020] Pascal Bercher and Conny Olz. $\mathrm{POP} \equiv \mathrm{POCL}$, right? Complexity results for partial order (causal link) makespan minimization. In Proc. of AAAI, 2020.

[Berg et al., 2019] Jeremias Berg, Emir Demirovic, and Peter J. Stuckey. Core-boosted linear search for incomplete MaxSAT. In Proc. of the 16th International Conference on the Integration of Constraint Programming, AI, and Operations Research, pages 39-56, 2019.

[Brachman and Levesque, 2004] Ronald Brachman and Hector Levesque. Knowledge Representation and Reasoning. Morgan Kaufmann Publishers, USA, 2004.

[Fox and Long, 1999] Maria Fox and Derek Long. The detection and exploitation of symmetry in planning problems. In Proc. of IJCAI, pages 956-961, 1999.

[Joslin and Roy, 1997] David Joslin and Amitabha Roy. Exploiting symmetry in lifted CSPs. In Proc.of AAAI, page 197-202, 1997.

[Kambhampati and Kedar, 1994] Subbarao Kambhampati and Smadar Kedar. A unified framework for explanationbased generalization of partially ordered and partially instantiated plans. Artificial Intelligence, 67(1):29-70, 1994.

[Kambhampati and Nau, 1996] Subbarao Kambhampati and Dana S. Nau. On the nature and role of modal truth criteria in planning. Artifical Intelligence, 82(1-2):129-155, 1996.

[Korhonen et al., 2017] Tuukka Korhonen, Jeremias Berg, Paul Saikko, and Matti Järvisalo. MaxPre: An extended MaxSAT preprocessor. In Proc. of the 20th Int. Conference on Theory and Applications of Satisfiability Testing, volume 10491 of LNCS, pages 449-456. Springer, 2017.

[Lifschitz, 1987] Vladimir Lifschitz. On the semantics of STRIPS. In Michael Georgeff and Amy Lansky, editors, Reasoning about Actions and Plans, pages 1-9. Morgan Kaufmann, San Mateo, CA, 1987.

[Lipovetzky and Geffner, 2017] Nir Lipovetzky and Hector Geffner. Best-first width search: Exploration and exploitation in classical planning. In Proc. of AAAI, pages 35903596, 2017.

[Muise et al., 2016] Christian Muise, J. Christopher Beck, and Sheila A. Mcllraith. Optimal partial-order plan relaxation via MaxSAT. Journal of Artificial Intelligence Research, 57:113 - 149, 2016.

[Nguyen and Kambhampati, 2001] XuanLong Nguyen and Subbarao Kambhampati. Reviving partial order planning. In Proc. of IJCAI, pages 459-464, 2001. 
[Olz and Bercher, 2019] Conny Olz and Pascal Bercher. Eliminating redundant actions in partially ordered plans A complexity analysis. In Proc. of ICAPS, pages 310-319, 2019.

[Pochter et al., 2011] Nir Pochter, Aviv Zohar, and Jeffrey S. Rosenschein. Exploiting problem symmetries in statebased planners. In Proc. of AAAI, 2011.

[Richter and Westphal, 2010] Silvia Richter and Matthias Westphal. The LAMA planner: Guiding cost-based anytime planning with landmarks. Journal of Artificial Intelligence Research, 39(1):127-177, 2010.

[Riddle et al., 2016] Pat Riddle, Jordan Douglas, Mike Barley, and Santiago Franco. Improving performance by reformulating PDDL into a bagged representation. In Proc. of the 8th Workshop on Heuristics and Search for Domainindependent Planning, pages 28-36, 2016.

[Rintanen, 2003] Jussi Rintanen. Symmetry reduction for SAT representations of transition systems. In Enrico Giunchiglia, Nicola Muscettola, and Dana S. Nau, editors, Proc. of ICAPS, pages 32-40, 2003.

[Rintanen, 2010] Jussi Rintanen. Heuristics for planning with SAT. In David Cohen, editor, Proc. of $C P$, pages 414-428, 2010.

[Say et al., 2016] Buser Say, André A. Ciré, and J. Christopher Beck. Mathematical programming models for optimizing partial-order plan flexibility. In Proc. of ECAI, pages 1044-1052, 2016.

[Shlyakhter, 2007] Ilya Shlyakhter. Generating effective symmetry-breaking predicates for search problems. Discrete Applied Mathematics, 155(12):1539-1548, 2007.

[Siddiqui and Haslum, 2012] Fazlul Hasan Siddiqui and Patrik Haslum. Block-structured plan deordering. In Proc. of the 25th Australasian Joint Conference on Advances in Artificial Intelligence, pages 803-814, 2012.

[Waters et al., 2018] Max Waters, Bernhard Nebel, Lin Padgham, and Sebastian Sardiña. Plan relaxation via action debinding and deordering. In Proc. of ICAPS, pages 278-287, 2018.

[Weld, 1994] Daniel S. Weld. An introduction to least commitment planning. AI Magazine, 15(4):27-61, 1994. 